\title{
Controlling human fixed-interval performance with fixed-ratio responding or differential reinforcement of low-rate responding in mixed schedules
}

\author{
HAROLD WEINER \\ Division of Clinical Training and Research \\ Saint Elizabeths Hospital, NIMH, Washington, D.C. 20032
}

Low response rates and postreinforcement pauses occurred under an FI schedule when a DRL schedule and the FI schedule operated in alternation. High constant FI responding (no pauses) occurred when FR and FI schedules alternated. These results were similar to previous ones which were obtained when FR or DRL responding occurred prior to exposure to an FI schedule.

Ss responding at high constant rates without postreinforcement pauses under a fixed-ratio (FR) schedule of reinforcement tend to maintain such responding when shifted to a fixed-interval (FI) schedule. Low response rates and postreinforcement pauses are obtained under FI schedules when Ss have previously produced such responding under a differential reinforcement of low rate (DRL) schedule. Responding under an FR schedule does not persist to affect final performances under a DRL schedule, nor does DRL responding determine final response rates and patterns under an FR schedule (Weiner, 1964, 1969).

The purpose of the present experiment was to replicate and extend these findings to conditions where performances under FR, DRL, and FI schedules are acquired and maintained more contemporaneously in time. Mixed schedules were employed to accomplish this purpose. A mixed schedule "is a compound schedule in which two or more component schedules operate in alternation, all in the presence of the same stimulus [Catania, 1968, p. 339]." In this experiment, performances under mixed DRL FI, mixed FR FI, and mixed DRL FR schedules were examined.

\section{SUBJECTS}

Two adult humans, one 44-year-old male (S 708) and one 50-year-old female (S 709), served as Ss. Both Ss were paid $\$ 3$ per 1-h experimental session, irrespective of their operant performances.

\section{PROCEDURE}

Each $S$ sat alone in a chair facing a five-digit electronic counter and $a$ microswitch button. The $\mathrm{S}$ began each experimental session with five zeros showing on the counter and was instructed to use the microswitch button to score as many points as possible. Addition of points depended on buttonpressing. An effective buttonpress required a force of approximately $20 \mathrm{~g}$ through a distance of $1 \mathrm{~cm}$ to close a microswitch mounted underneath it.

At the beginning of the first experimental session, the following instructions were read to each $\mathbf{S}$ : "Your task is to score as many points as possible on this counter [E indicated]. You can do this with this button. You will be paid $\$ 3$ for each 1 hour session in this room."

After the instructions, Ss were conditioned under the following progression of two-component mixed (mix) schedules: mix FI 10-sec DRL 10-sec, mix FI 10-sec FR 80, and mix DRL 10-sec FR 80. Under the FI 10-sec component, a reinforcement (100-point addition to the counter score) was provided for a single response if at least $10 \mathrm{sec}$ had elapsed from a preceding reinforcement. All responses before 10 sec had elapsed had no effect on the occurrence of reinforcement. Under the DRL 10 -sec component, a 100-point reinforcement was contingent on the spacing of two successive responses by at least $10 \mathrm{sec}$. Interresponse times of less than $10 \mathrm{sec}$ postponed reinforcement for at least 10 sec. Finally, under the FR 80 component, a 100-point reinforcement was delivered each time $S$ pressed the response button 80 times.

The components of each of the three mixed schedules alternated with every reinforcement, without any discriminative stimuli. Under the mix FI 10-sec DRL 10-sec schedule, both Ss began each session with the FI 10-sec component. Under the mix FI 10-sec FR 80 schedule, S 708 began each session under the FR 80 component, while S 709 started each session under the FI 10-sec component. For both Ss under the mix DRL 10-sec FR 80 schedule, a session began with either the FR 80 or the DRL 10-sec component.
The Ss received 101 -h conditioning sessions under each of the three mixed schedules. Responses were recorded on c um u lative recorders and electromechanical counters.

$$
\text { RESULTS }
$$

Figure 1 summarizes the performances of the Ss under the three mixed schedules. Under the mix FI 10-sec DRL 10-sec schedule, both Ss began the first session by responding at a high constant rate. Even though such high constant responding was reinforced initially under the FI component, Ss produced low-rate performances (low rates of responding with postreinforcement pauses) under the FI component during the first session as low-rate performances developed under the DRL component. Low-rate performances were maintained under the FI 10-sec and DRL 10-sec components of the mix FI 10-sec DRL 10-sec schedule throughout Sessions 2-10 (Sessions 2-9 not shown).

Both Ss responded at low rates and showed extended pausing at the beginning of the 1st session under the mix FI 10-sec FR 80 schedule. Some FI reinforcements followed pauses during the 2nd session for S 708 and throughout the first 4 sessions for S 709. Nevertheless, except for the initial moments of the 1st and 2nd sessions, S 708 gave stable high-rate performances (high rates of responding without postreinforcement pauses) under the FI 10-sec and FR 80 components throughout all 10 sessions of the mix FI 10-sec FR 80 schedule (Sessions 4-9 not shown). S709 showed some high-rate performances until after the first $10 \mathrm{~min}$ of the 4 th session. During the final 50 min of the 4 th session and throughout Sessions 5-9 (not shown), S709 maintained the high-rate performance shown for the 10th session. As in the 10th session, S 709 always paused prior to the first FI reinforcement during Sessions 5-9.

A similar response pattern was obtained from both Ss at the beginning of the first session of the mix DRL 10-sec FR 80 schedule. An FR 80 reinforcement was followed by high constant rates for a period of time under DRL 10-sec, while a DRL 10-sec reinforcement was followed by extended postreinforcement pausing under FR 80. However, after approximately 30 min of the first session, this pattern disappeared and both Ss gave high-rate performances under the FR 80 component and low-rate performances under the DRL 10-sec component. Such high- and low-rate performances were maintained during Sessions 2-10 (Sessions 2-9 not shown) of the mix 

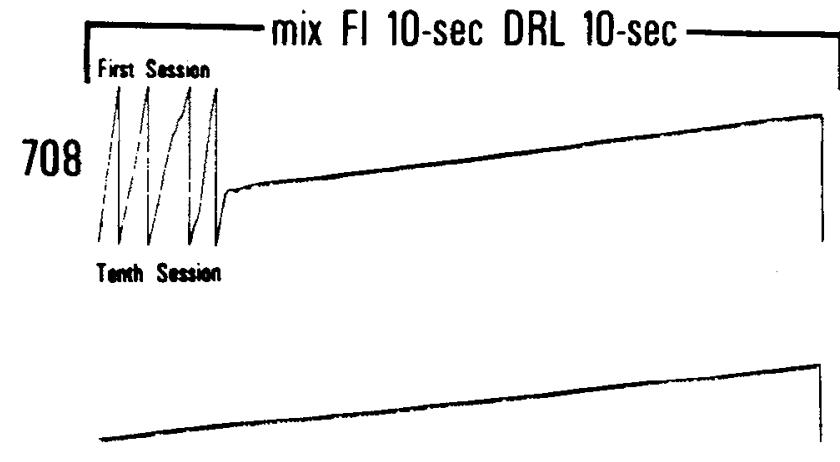

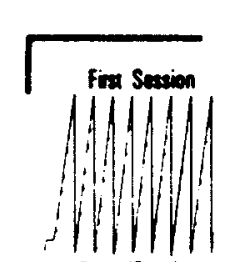

Fim 15 min

Tomon Servion
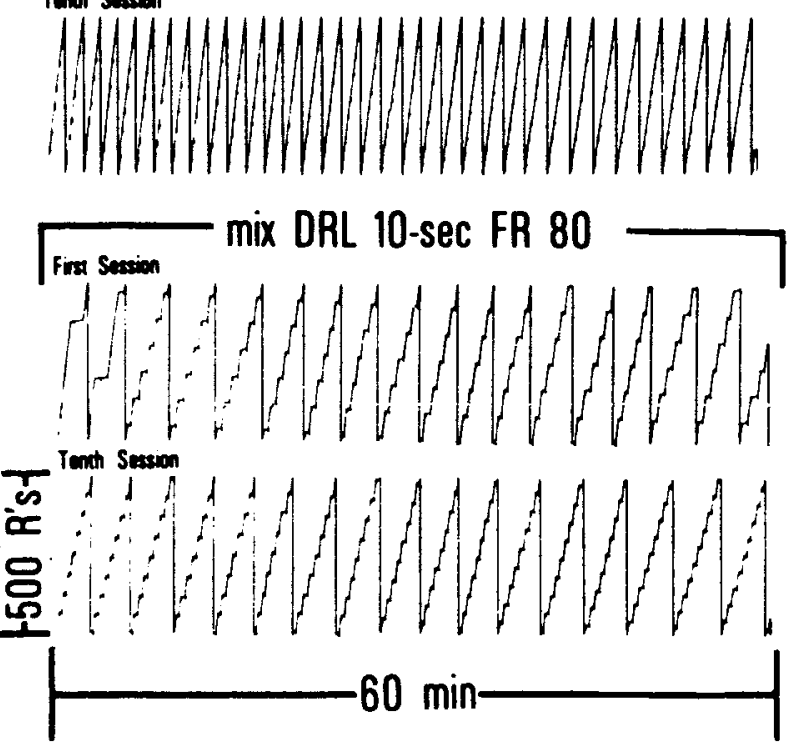

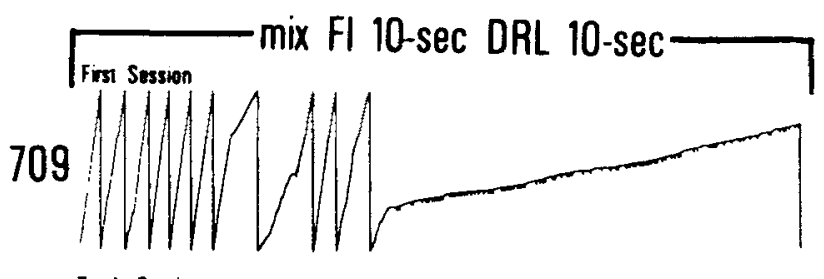

Tenth Session
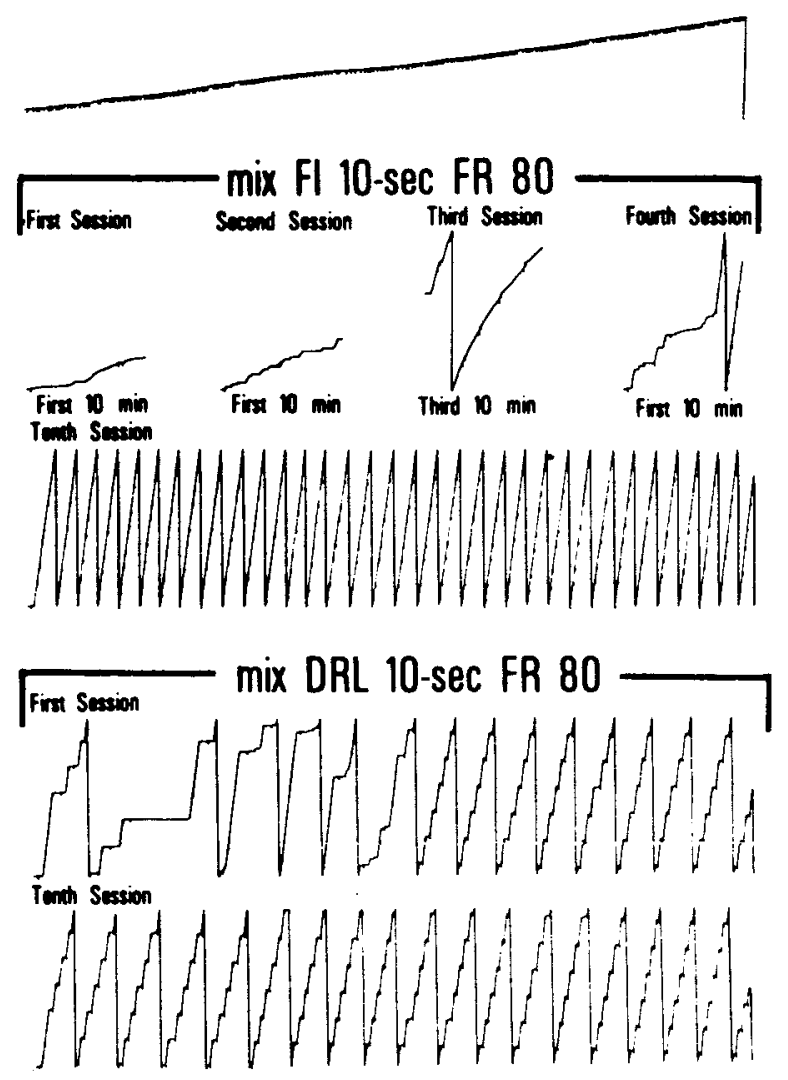

Figure 1

DRL 10 sec FR 80 schedule. As happened under the mix FI 10-sec FR 80 schedule, S 709 always paused at the beginning of each session under the mix DRL 10-sec FR 80 schedule.

Both low- and high-rate performances are commonly obtained under a simple FI 10-sec schedule with humans (Weiner, 1969). The present experiment has demonstrated that such performances can be produced experimentally and hence controlled by alternating DRL 10 sec or FR 80 components with FI $10-\mathrm{sec}$ in mixed schedules. Thus, DRL 10-sec responding generated low-rate FI 10-sec performances under the mix FI 10-sec DRL 10-sec schedule, while FR 80 responding produced high-rate FI 10-sec performances under the mix FI 10-sec FR 80 schedule.
Whereas DRL 10-sec and FR 80 responding affected FI 10-sec responding, DRL 10 -sec and FR 80 responding did not affect each other under the mix DRL $10 \mathrm{sec}$ FR 80 schedule. This suggests that DRL 10 -sec and FR 80 effects were maintained under FI because FI provided no detrimental consequences for such maintenance.

Findings similar to those obtained in the present experiment have been reported previously (Weiner, 1964, 1969) in experimental paradigms where Ss were exposed to DRL or FR prior to FI or prior to one another and when Ss had more extensive training $(10 \mathrm{~h})$ under FR or DRL prior to FI. Apparently, FR and DRL responding can exert their differential effects on FI performance despite different amounts of training under FR and DRL and irrespective of whether or not FR and DRL responding is occurring when FI performance is being established.

\section{REFERENCES}

CATANIA. A. C. Contemporary research in operant behavior. Glenville, $\mathrm{nl}$ : Scott, Foresman, 1968.

WEINER, $H$. Conditioning history and human fixed-interval performance. Journal of the Experimental Analysis of Behavior, 1964, 7, 383-385.

WEINER, H. Controlling human fixed-interval performance. Joumal of the Experimental Analysis of Behavior, 1969 , 12, 349-373. 